\title{
Informed consent: time for more transparency
}

Yusuf Yazici*1 and Hasan Yazici

\begin{abstract}
Informed consent is not only for documenting a patient's acceptance of enrolling in a clinical trial. It currently is the patient's and, we propose, should also be the public's main source of information regarding the reasons for the planned study, what is known in the field about the proposed trial, and what to expect as far as efficacy and harm. Informed consent is not currently part of the clinical trial registries. For purposes of full disclosure to the patients and the public, the informed consent should be part of the required documents for such registries.
\end{abstract}

The World Health Organization has developed a single international standard for the information that authors of clinical trials must disclose [1]. The informed consent form (original or subsequent versions if the trial protocol necessitates) is not among the listed items. More recent schemes to expand registration of clinical trials also do not include full disclosure of informed consent forms $[2,3]$. We strongly feel that the exclusion of informed consent is a serious omission in our current attempt to make clinical trials more transparent; we give four specific reasons in support of this view:

1. The patients and the public should know whether the study involves a medication that has already been shown to be effective in other similar studies. Informed consent forms are supposed to include this information to help patients make an informed decision about the possible benefit they may get from participating in the trial. Although all clinical trials should ideally be conducted with the goal of improving medical knowledge, other reasons for clinical trials, including simple promotion, are seen [4]. There is little scientific or societal gain in repeating clinical trials with medications that have already shown efficacy [5].

*Correspondence: yusuf.yazici@nyumc.org

'New York University School of Medicine, NYU Hospital for Joint Diseases,

246 East 20th Street, New York, NY 10003, USA

Full list of author information is available at the end of the article
2. There can be considerable variation in how informed consent is addressed by investigators from different cultures and socioeconomic settings, even within the same multinational trial [6]. These differences can become more important and worrisome when drug trials are conducted in developing countries [7] where the investigators and sponsors might be more relaxed as to ethical standards of human experimentation. Transparency of the informed consent forms may facilitate the local and international surveillance of unethical scientific conduct.

3. There are examples of clinical trials that appear unnecessarily prolonged after the evidence is already available for a clear beneficial effect. We had expressed such concern for a major drug trial in rheumatology some years ago [8]. In that instance, we had pointed out that a beneficial effect of the study drug was apparent at the end of the first year, leading to a concern regarding whether patients who were invited to continue the trial into the second year were informed that there was a significant chance they would not get the superior therapy during the second year. Our concerns would have been addressed right away had the informed consent for the extension been available in a clinical trials database.

4. The issue of informed consent is even more important in trials designed to assess safety. If one takes the Popperian view that an honest attempt at falsification is the correct way to test a hypothesis [9], a randomized clinical trial to assess safety is difficult to design and conduct. A rigorous safety study includes an intention to bring harm. The medication will be considered safe if the null hypothesis cannot be documented concerning the intended harm. One might even suggest that a societal good can hardly ever be justified in a randomized controlled safety trial [10]. Therefore, the wording of the informed consent document is especially important in such safety trials.

A recent communication addressed the issue of informed consent related to such a trial in some detail [11]. The principal investigator of this randomized controlled safety trial stated that he was unsure whether he was able to disclose the informed consent forms of this trial. How does one inform a patient that the scientific experiment 
involves deliberate harm, as all safety trials to some degree potentially do, as an endpoint?

The concept of clinical trial registry has been designed to disseminate knowledge about clinical trials. Currently, brief protocol summaries, including aims, primary outcomes, inclusion and exclusion criteria, duration of the trial, and planned intervention, of all clinical trials are available on the internet. Concern for proprietary rights precludes a verbatim openness of the drug protocols at this time. This can be understandable. What is not understandable is why the informed consent forms, basically the translation of what the protocol dictates to the patient level, is not part of these registries.

It is worth noting that the current practice of approving the clinical trial applications, including the informed consent forms, at institutional review boards (IRBs) does involve the input of the public. There is at least one layman member, from the local community, who is part of the IRB. However, this is a very limited and short-term public availability and thus a very limited and short-term transparency. What needs to be done is to make this transparency widespread and permanent, thus greatly enhancing the awareness of the public, peers, and patients of what is being studied.

We propose that, from now on, all clinical trial investigators be required to post the informed consent documents in public clinical trial registries. Thus, all interested parties could easily review the initial informed consent form as given to the potential trial participants (or forms in case of multicenter trials due to separate local IRBs) and any amendments thereafter.

We appreciate that the transparency we are proposing might not be the answer in finding a solution, especially in the short term and in differing geographic locations, to each of the four separate issues we listed above. However, we strongly suggest that it will be a step in the right direction. For example, it simply is not practical that the public at large should have a say in the decision of a local IRB before or after each time a decision is reached for any one trial. On the other hand, the wide recognition that anyone concerned can have full access to the mode of conduct of a trial before, during, or after it is conducted will, we like to think, eventually lead to higher ethical standards. Our proposal is akin to the now wellrecognized 'What would my patients or colleagues think?' yardstick to improve physician-industry relations [12].

Since the main purpose of what we propose is availability upon need and since all data deposit will most likely be electronic, such storage would be relatively easy. In such a scheme, it is especially important to leave the older versions of the amended forms in the registry. Only then can a peer-reviewer or any other individual auditor assess whether the trial is running, has been running, or had been running in accordance with the best scientific and ethical conduct.

We, like others [4], are concerned about erosion of trust in physicians. Our relationship with the pharmaceutical industry, including designing and running drug trials, is an important component of this issue. We like to think that total transparency of the patients' informed consent forms would provide a significant step in restoring trust. The public's informed consent to what we do in a clinical trial will not be complete unless the patients' informed consent document becomes public.

Abbreviation

IRB, institutional review board.

\section{Competing interests}

YY has served as a consultant for Bristol-Myers Squibb Company (Princeton, NJ, USA), Celgene Corporation (Summit, NJ, USA), Centocor, Inc. (Horsham, PA, USA), Genentech, Inc. (South San Francisco, CA, USA), Roche (Basel, Switzerland), and UCB (Brussels, Belgium). HY declares that he has no competing interests

\section{Author details}

${ }^{1}$ New York University School of Medicine, NYU Hospital for Joint Diseases, 246 East 20th Street, New York, NY 10003, USA. 2University of Istanbul, Cerrahpasa School of Medicine, Fatih, Istanbul, 34098, Turkey.

Published: 3 June 2010

\section{References}

1. Watts R, Cubie S: Clinical trial registration. Rheumatology 2008 , 47:1595-1596.

2. Summerskill W, Collingridge D, Frankish H: Protocols, probity, and publication. Lancet 2009, 373:992.

3. Strahlman E, Rockhold F, Freeman A: Public disclosure of clinical research. Lancet 2009, 373:1319-1320.

4. Kramer BS, Wilentz J, Alexander D: Getting it right: being smarter about clinical trials. PLoS Medicine 2006, 3:7560-7561.

5. Sox HC, Rennie D: Seeding trials: just say 'no'. Ann Intern Med 2008, 149:279-280.

6. Rikkert MG, Lauque S, Frölich $L$ : The practice of obtaining approval from medical research ethics committees: a comparison within 12 European countries for a descriptive study on acetlycholinesterase inhibitors in Alzheimer's dementia. Eur J Neurol 2005, 12:212-217.

7. Shapiro HD, Meslin EM: Ethical issues in the design and conduct of clinical trials in developing countries. N Engl J Med 2001, 345:139-142.

8. Yazici Y, Yazici H: Trial of etanercept and methotrexate with radiographic and patient outcomes two-year clinical and radiographic results: comment on the article by van der Heijde et al. Arthritis Rheum 2006, 54:3061-3062.

9. Heath I: Dare to use your own intelligence. BMJ 2008, 337:a1319.

10. Yazici H: Use and abuse of the controlled clinical trial. Bull NYU Hosp Jt Dis 2007, 65:132-134.

11. Lenzer J: Truly independent research? BMJ 2008, 337:a1332.

12. Coyle SL: Physician-industry relations. Part 1: individual physicians. Ann Intern Med 2002, 136:396-402.

doi:10.1186/ar3004

Cite this article as: Yazici Y, Yazici H: Informed consent: time for more transparency. Arthritis Research \& Therapy 2010, 12:121. 